Research Article

\title{
College Physical Education Teaching Aided by Virtual Reality Technology
}

\author{
Junniao Meng \\ Department of Physical Educaton, Zhengzhou Shengda University, Zhengzhou 450000, Henan, China \\ Correspondence should be addressed to Junniao Meng; 100918@shengda.edu.cn
}

Received 22 July 2021; Revised 27 August 2021; Accepted 24 September 2021; Published 18 October 2021

Academic Editor: Sang-Bing Tsai

Copyright (c) 2021 Junniao Meng. This is an open access article distributed under the Creative Commons Attribution License, which permits unrestricted use, distribution, and reproduction in any medium, provided the original work is properly cited.

In recent years, College PE has received more and more attention. Traditional PE and teaching cannot meet the demand of students in today's society and cannot attract students' interest in sports. Under the current situation that the country actively promotes information science education, there is an urgent need to innovate PE teaching methods. VRT is very important to support college PE. In order to improve the quality of university PE and increase students' interest in sports, this article uses the Internet of Things as a research background and $5 \mathrm{G}$ technology as a research topic. It provides technical support for the Internet of Things and research sports colleges on the Internet of Things. This study builds a virtual sports teaching environmental education through VRT. Experimental data were collected by sensors and other types of infrastructure. The experiment counted the number of sports safety incidents that occurred before and after the application of VRT in five universities of A, B, C, D, and E, collected and analyzed the teaching data of PE teachers in five universities, and studied the changes in student learning and interest in sports. These experimental data are used to analyze the impact of the application of VRT in PE on sports safety, PE teachers, and students. The final result shows that, after applying VRT in PE, the five schools have reduced the number of safety incidents in sports by more than $65 \%$. VRT has the greatest impact on the teaching philosophy of PE teachers and the least impact on teachers' teaching management. After the application of VRT, the number of students who independently engage in PE and sports has increased by more than $20 \%$, and the students' interest in sports learning and sports has increased. Finally, the application of VRT in the PE of the five schools accounted for $25 \%, 21 \%, 11 \%, 35 \%$, and 30\%, respectively. The application of VRT will also indirectly affect the quality of PE in universities.

\section{Introduction}

1.1. Background Meaning. In order to respond to the call of the country to allow future young people to go out of the classroom, out of the dormitory, to the sun, on the campus, and on the playground, so as to improve the physical fitness and endurance of students, outdoor sports are widely carried out in schools at all levels across the country. The school requires students to complete a certain amount of exercise every semester, and the long-distance running performance will also be an integral part of the sports performance. Faced with these changes, PE in colleges and universities has gradually made a change, the traditional mode of teaching $\mathrm{PE}$ is gradually being abandoned, and PE began to change direction toward intelligent form. Universities have begun to use IoT technology to process sports-related information.
Sensors and other smart devices are used to perceive and detect students' sports status and physical fitness information and use the Internet of Things technology and VRT to control various smart devices to assist students in reasonable and healthy sports.

Virtual PE actually helps to improve the quality of sports and technology students' interest in sports through VRT. Applying the Internet of Things and VRT to PE classes can help students do some physical exercises independently and can upload relevant sports teaching videos to smart devices to achieve assisted teaching for students, thereby reducing PE teachers' teaching burden. On the other hand, using the Internet of Things and VRT to assist students in sports education can provide correct and scientific sports guidance to students and tailor-made sports programs and plans that suit them to ensure that the safety 
of student sports can reduce the occurrence of sports safety incidents and reduce the hidden dangers of sports safety. The Internet of Things and VRT have made certain contributions to promote the development of PE, which can make PE and teaching innovation in science and technology [1].

1.2. Related Work. Many scholars have conducted related researches on PE teaching in colleges and universities. Yang analyzed and researched the "student physical condition" and "teaching condition" and made some suggestions for the reform of university PE [2]. Zhang analyzes the positive influence of "Internet plus education" on the concept of PE, the form of teaching, and the mode of communication, builds a sports platform, and applies a $3 \mathrm{D}$ mixed teaching model and a diversified target assessment system to explore the reform of university sports in the context of educational information [3]. Wei-Ping et al. used literature and logical analysis to divide the college physical education curriculum reform into three stages: accumulation stage, differentiation stage, and deepening stage. They sorted out the theoretical and practical results of each stage of the college physical education curriculum and rethinked the positioning and direction of the college physical education curriculum reform in the new era [4]. Stormoen combines the main points of the theory with reality to study the factors that promote the positive experience of $\mathrm{PE}$ in colleges and universities [5]. However, these scholars still have some problems in the research process. Some of Yang's views are too old-fashioned; Zhang's experimental process is too cumbersome; Wei's argument does not accord with the argument; Stormoen's conclusion is too hasty.

1.3. Innovation in this Article. This article takes VRT as the research object of the Internet of Things sports network, uses the $5 \mathrm{G}$ network as the technical support of the Internet of Things environment, and analyzes the VRT in sports universities, sports safety, and PE teachers and students. The innovation of this work is mainly reflected in the following aspects. (1) This research is carried out in the Internet of Things environment, which provides a network environment for collecting experimental data, applying experimental techniques, and conducting experiments experiment. More convenient, (2) the application of VRT in sports schools has innovated the teaching methods of sports schools and brought more benefits to sports schools. (3) The experiment was analyzed from the perspective of teachers and students, and five universities were used as research objects to ensure the validity of the data.

\section{Relevant Techniques for Teaching Research on PE in Colleges}

2.1. Virtual Reality Technology. VRT is an innovative manmachine exchange technology. Through docking control equipment, computer programs are used to process and calculate the information in the scene and finally display the processed information on the terminal device to form a real scene similar to the three-dimensional environment; in this environment, people will have the sense of immersion $[6,7]$. VRT integrates multimedia, computer graphics, network detection, and sensor recognition. Its application speed in various fields of our daily life is unprecedented and its application range is extremely wide [8]. VRT uses scene drawing and other related technologies to create a virtual environment similar to the real environment and allows users to interact with objects in the virtual environment through sensor devices $[9,10]$. The wireless sensor can also be wirelessly charged to achieve long-term recording, so the sensor can maintain stable signal transmission within a year. VRT is immersive, interactive, and imaginative. With the advancement of technology, people's lifestyles have become more and more convenient and fast, and intelligent services have replaced people with most of the things in their daily lives.

The early VRT is very different from the current VRT. The prototype of the early VRT is to describe things that do not exist. The current VRT is realized through networks and devices. Later, after the development of the times, for a period of time in the west, hunted animals were preserved in the form of animal and plant specimens, and virtual and reality appeared in a true restoration. Still, later, people with a variety of platforms allow audiences to experience a similar feeling of time travel; this is the time and space to conquer the virtual reality generated; virtual reality development has thus been a major breakthrough. Until nowadays, the combination of VRT, network technology, and sensor equipment has once again achieved major technological breakthroughs [11]. VRT is widely used in aerospace, medicine, training, games, education, and other industries, and its development has brought technological breakthroughs to related research in various fields [12]. For example, virtual reality technology has brought unprecedented reforms to medical teaching. Through the simulation of realistic clinical scenes, students can feel personally in the simulation environment, thereby making surgical skills training more efficient.

2.2. Internet of Things. The Internet of Things is a complex of multiple fields, multiple disciplines, and a huge technical architecture. It is a product derived from the gradual development and improvement of the modern Internet. It is based on the Internet and connects all objects or people in the network environment so that people or things in the Internet of Things can exchange information and communicate with each other through this technology [13]. But there are also many differences between the Internet of Things and the Internet. The essence of the Internet of Things is perception and service, and the essence of the Internet is the push and sharing of online information and content based on mobile phones and PCs. Things are contiguous and the object, thereof, is a new technology of information occurring in the case of various types of sensors in combination with each other and the Internet; it may also be used as an information exchange technology; its essence is 
perceived services $[14,15]$. The Internet is a huge network connected in series between networks, and information exchange is interactive and real-time [16]. The cost of using the Internet for information exchange is lower, and it is easier to meet the individual needs of everyone. The Internet of Things is usually divided into three components: perception layer, network layer, and application layer. Through the Internet of Things, technology to communicate objects or people in the environment can achieve the positioning, detection, tracking, and related management functions of objects or people [17]. The task of the perception layer is to identify objects and collect relevant information in the system, so as to realize the knowledge and perception of "things." The network layer realizes the transmission, preliminary processing, classification, and aggregation of information through the existing Internet, radio and television network, communication network, etc., and is used to communicate between the perception layer and the application layer. The application layer is the purpose of the development of the Internet of Things. To implement these functions, the field of art involved things very much, which in many technical RFID sensor technologies and wireless networking technologies allows key technology systems. In the Internet of Things environment, the relevant information in the RFID tag can be identified by using the radio frequency signal emitted by the RFID technology, and finally, the remote automatic identification and inspection of the item can be realized. Wireless sensors have a lot of room for development in the Internet of Things environment, mainly in the process of information collection, transmission, perception, and self-organization, and they have a certain degree of stability. As society's demand for wireless communication continues to increase, the Internet of Things has emerged, which has greatly promoted the rapid development of wireless communication and has become more and more important in sensors [18]. The development and application of the Internet of Things are not only reflected in the development of science and technology but also reflected in the PE of modern universities.

The emergence of 5G technology brings great development prospects for the Internet of Things. The application conditions of the Internet of Things in wireless networks are very demanding, it must meet a large number of connections, and the network delay requirements are particularly short, but most of the current wireless networks cannot meet the demanding conditions of the Internet of Things applications. It appears that the $5 \mathrm{G}$ network for use on the wireless network has provided technical support for the Internet of Things, which is supported by the application of things about aspects. (1) The 5G network architecture exists in the form of network slicing and architecture layering, creating exclusive virtual networks for various fields according to various requirements [19]. (2) 5G deployed in a network environment, a number of base stations, real-time control, and coordination of the interference and frequency bands for a variety of standard formats and by using a dynamic adaptive Mesh access network. (3) The 5G network analyzes a large amount of network data in the Internet of Things by integrating big data, so as to make reasonable plans for various fields and provide services for various users [20]. 5G and the Internet of Things compete and complement each other. The Internet of Things needs a $5 \mathrm{G}$ network to realize its own network framework and connect various devices in the network environment. 5G network also needs to promote its own technological progress through the development of the Internet of Things and constantly meet the needs of various fields $[21,22]$.

2.3. $P E$. $P E$ is a kind of social activity, and each is a specialized subject in the school. Sports can provide the needs of production and life of people in the development of human society, in accordance with the law of the quality of people's physical and psychological, to enhance physical fitness by strengthening exercise on the body and improve their motor function $[23,24]$. Sports play an important role in people's moral cultivation, and it can also promote the development of social culture. PE teaching is an educational process with strong purpose, planning, and organization. This process requires the joint participation of teachers and students to teach students sports knowledge and skills, improve their physical quality, and cultivate their strong willpower $[25,26]$. PE is an important part of higher education in China. It can bring the students the effect of strengthening their body and body, improve their physical and mental health and interpersonal communication ability, and play an important role in the overall development of students' morality, intelligence, and physical and physical beauty [27, 28].

With the popularization of the Internet of Things and wireless communication technology, a large amount of information is stored on the network. The powerful processing ability of sensors and computers is used to process this information, and the analysis results are obtained by comparing with database and information, so as to make sports information more intelligent. The college PE teaching combined with the Internet of Things is also called intelligent sports. It uses the Internet of Things technology to sense the students' sports situation through sensors and connects to the intelligent terminal to analyze the data and fully understand the athletes' sports conditions. The key technologies involved in the field of the Internet of Things are RFID technology, sensor technology, wireless network technology, human intelligence technology, and cloud computing technology. Through these technologies, we can capture reliable data, transmit it safely, and obtain all aspects of athletes' information. With a good smart sports infrastructure, there should also be a service provider to improve and explore the future possibilities of the facility. These organizations are responsible for working with athletes and providing low-cost, high-performance, and intelligent services to promote the overall development of the sport.

\section{Experimental Design of PE Teaching Research in Colleges and Universities Based on VRT}

3.1. Data Acquisition. We collected experimental data from 5 schools and counted students' interest in virtual technology, changes in the application of virtual technology to 
teaching, and students' views on the application of virtual technology to teaching. Finally, the sports teaching mode adopted by five colleges and universities was counted, and the application of VRT in five colleges and universities was analyzed.

3.2. Implementation of $5 G$ Technology. The important application scenarios of $5 \mathrm{G}$ in the future are inseparable from the mobile Internet and the Internet of Things. The cellular network provides a wide range of access services to meet the rapid development requirements of the mobile Internet and the Internet of Things. Among them is low-density code technology, which is a special nonorthogonal multiple access field code that can use sparse dispersion spectrum, which is mainly expressed as follows:

$$
L_{k}(n)=x_{k} * f_{k}(n), \quad n=1,2, \ldots, N .
$$

In the formula, $x_{k}$ represents the code word to be spread, and $f_{k}(n)=\left[f_{k}(1), f_{k}(2), \ldots, f_{k}(N)\right]^{T}$ represents the spreading sequence. Since some elements in the spreading codeword of the low-density code technology are zero, the code word exhibits sparse characteristics. The received signal model of low-density coding technology can be expressed as

$$
s=G_{x}+n,
$$

where $s$ represents the received signal, $G=\left[g_{1}, g_{2}, \ldots, g_{K}\right]$ represents the sparse spread spectrum matrix, and $n$ represents a Gaussian white noise vector normalized with zero variance. The position of no 0 elements in the sparse matrix Gis generally determined by the low-density spread spectrum indicator matrix $H$. A $6 \times 9$ low-density spread spectrum indicator matrix is expressed as

$$
\begin{array}{lllllllll}
0 & 0 & 1 & 0 & 0 & 0 & 1 & 0 & 1 \\
0 & 1 & 0 & 1 & 0 & 0 & 1 & 0 & 0 \\
0 & 0 & 1 & 1 & 0 & 1 & 0 & 0 & 0 \\
1 & 0 & 0 & 0 & 1 & 0 & 0 & 0 & 1 \\
1 & 0 & 0 & 0 & 0 & 1 & 0 & 1 & 0 \\
0 & 1 & 0 & 0 & 1 & 0 & 0 & 1 & 0
\end{array} .
$$

Each column of the matrix represents the spreading indicator vector of a data layer; " 1 " indicates the position of filling nonzero elements in the sparse spread spectrum matrix $G$; " 0 " indicates the position of the zero-filled element in the sparse spreading moment $G$.

Sparse code division multiple access (SCMA) is a code domain nonorthogonal multiple access technologies developed from low-density code technology. The encoder of sparse code division multiple access can be defined as

$$
q: B^{\log _{2}(M)} \longrightarrow X,
$$

where $B$ is the bitstream, $X$ is the $K$-dimensional sparse code word, and $M$ is the code-book size. The encoder of sparse code division multiple access is a mapping of each $\log _{2}(G)$ bit to a $K$-dimensional sparse code word. The design of the SCMA codebook directly affects the performance of the
SCMA system. An abstract SCMA code-book structure can be expressed as

$$
T^{*}, R^{*}=\operatorname{argmax}_{T, R} d(\eta(T, R, J, S, N, K)) .
$$

In formula (5), $d$ is the SCMA code-book design criterion; $T$ is a mapping matrix; $R$ is the multidimensional constellation; $J$ is the number of system users; $S$ is the size of the code-book set; $N$ is the number of nonzero elements in the codeword; $K$ is the code dimension word.

The message transmission principle of 5G technology: suppose that $I_{x \rightarrow f}(x)$ represents the message transmitted from variable node $x$ to functional node $f$, and $I_{f \rightarrow x}(x)$ represents the message transmitted from functional node $f$ to variable node $x$. The message update from variable node to function node is expressed as

$$
I_{x \longrightarrow f}(x)=\prod_{h \in n(x) /\{f\}} I_{h \longrightarrow x}(x) .
$$

The message passing update from function node to variable node is expressed as

$$
I_{f \longrightarrow x}(x)=\sum_{\{x\}} f(X) * \prod_{y \in n(f) /\{x\}} I_{y \longrightarrow f}(y),
$$

where $X=n(f)$ represents the set of independent variables of the function $f, h$ represents all the function nodes connected to the variable node $d$, and $y$ represents all the variable nodes connected to the function node $r$.

The 5G system mainly includes three aspects, namely, the speed demand, the delay demand, and the energy cost demand. Among the three demands, the most urgent one is the speed demand. The speed demand here mainly refers to the communication demand, because we are now in the communication era. The relevant formula is expressed as

$$
\frac{\text { bits }}{\mathrm{s} \cdot \mathrm{km}^{2}}=\frac{\text { cells }}{\mathrm{km}^{2}} \times \frac{\text { bits }}{\mathrm{s} \cdot \mathrm{Hz} \cdot \text { cell }} \times \mathrm{Hz} .
$$

The low-latency and high-reliability technology improves the reliability of data transmission through error correction and retransmission, adopts maximum likelihood decoding, and performs a weighted combination of soft decisions on data packets. Let $n$ be the number of retransmissions of the data packet, let $n_{\max }$ be the maximum number of transmissions, and $1 \leq n \leq n_{\max }$, weighted by the signal-to-noise ratio, to obtain the combined data:

$$
D(t)=\sum_{i=1}^{n} d_{i}(t) \omega_{i}
$$

where $d_{i}(t)$ represents the data received at the $i$ time, and $\omega_{i}$ represents the normalized signal-to-noise ratio received at the $i$ transmission. The normalized signal-to-noise ratio is the ratio of the energy per bit signal $E_{b}$ to the noise unilateral power spectral density $n_{0}$. In an additive white Gaussian noise channel with a standard deviation of $\sigma, \omega_{i}^{\prime}=1 / \sigma^{2}$; therefore, the normalized signal-to-noise ratio is 


$$
\omega_{i}=\frac{\omega_{i}^{\prime}}{\sum_{i=1}^{n} \omega_{i}^{\prime}}, \quad(i=1,2, \ldots, n) .
$$

Assuming that the conditions of each transmission process are the same, the signal-to-noise ratio received each time should be the same, then $\omega_{i}=1 / n$, and the above formula can be simplified to obtain the combined data packet as

$$
D(t)=\sum_{i=1}^{n} d_{i}(t) \omega_{i}=\frac{1}{n} \sum_{i=1}^{n} d_{i}(t) .
$$

\section{Data Analysis of College PE and Teaching Research under VRT}

4.1. Influence of VRT on the Safety of College PE. Security in college PE has been the focus of schools and parents were concerned; the occurrence of security incidents sports school education and teaching the sport had some negative impact. However, with the popularization of 5G VRT, the hidden danger of college sports safety has dropped significantly, and the frequency of sports safety incidents has decreased. This study studied the use of 5G VRT and the number of sports safety incidents in 5 universities of $A, B, C, D$, and $E$ and discussed the impact of $5 \mathrm{G}$ VRT on computer security. Reasonable influence compares and analyzes the status quo of PE. Table 1 lists the statistics of sports accidents.

From the data in the table, we can know that after VRT is used in physical education, college physical education has made great progress. Among them, the use of 5G VRT usage refers to the technology popularization $G$ of the university and the proportion of the number of classes $C$. The formula can be expressed as

$$
\text { usage }=\frac{G}{C} \times 100 \% \text {. }
$$

The reduced number dwindle numbers refers to the proportion of the reduced number of sports safety incidents after the use of 5G VRT to the number before the use of sports safety incidents that occurred before the technology is used. The formula can be expressed as

$$
\text { dwindle numbers }=\frac{\text { after use }- \text { before use }}{\text { before use }} \times 100 \% \text {. }
$$

In order to compare and analyze the changes in the number of sports safety incidents in five colleges and universities more intuitively, we replaced Table 1 with a graph to observe the changing trends of each series of data. The final result is shown in Figure 1.

According to the data and graph change trends in Figure 1, we can see that after the use of VRT, the number of college sports safety incidents has been significantly reduced, and the reduction is closely related to the use of VRT. School D's popularity of VRT has reached $35 \%$, and the number of sports safety incidents in this university has been reduced compared to the ratio of data before use, reaching $85 \%$. School C's popularity of VRT is relatively small, only $11 \%$, so the number of sports safety incidents in this college is also less than the proportion of data before use, only $65.1 \%$. This shows that VRT has a significant positive effect on the safety of college PE.

4.2. Influence of VRT on the Teaching of College PE Teachers. We select the teacher's teaching philosophy, teaching attitude, teaching content, teaching methods, teaching management, and teaching effect evaluation as the impact items of VRT; it is known that the total number of teachers in the five schools is $233,251,224,236$, and 242 , respectively. We count the number of teachers in each impact item and their proportions. The number of people counted is shown in Table 2, and the proportion table is shown in Table 3.

According to the data in Tables 2 and 3, we can see the changes that VRT can bring to the teaching of school PE teachers, but the proportion of teachers who have changed is not large. The percentage data in Table 3 is the ratio of the number of teachers $T_{i j}$ corresponding to $P$ in Table 2 to the total number of teachers $A_{i}$ in the school. $i$ corresponds to the school, and $j$ corresponds to the position of the influence item. The formula can be expressed as

$$
P=\frac{T_{i j}}{A_{i}} \times 100 \% \text {. }
$$

In order to compare the number of teachers in each impact item of the five schools more intuitively, we converted Tables 2 and 3 into graphs, and the conversion results are shown in Figures 2 and 3.

According to the data in Figures 2 and 3, we can see that the teaching mode of $\mathrm{PE}$ teachers in school $C$ is relatively less affected by VRT, and the school has $130 \%$ of the total number of impact items. School $A$ was most affected, and the total number of people in each affected item in this school was $151.5 \%$. The five schools have the least number of teachers in teaching management, and the total number of teachers is only $76.3 \%$. It can be seen that VRT has little effect on the teaching management of college PE.

4.3. Effect of VRT on Students' Physical Learning. The traditional PE teaching model can no longer meet the needs of students' PE so that students gradually lose interest in PE and sports. The emergence of VRT has changed the way of $\mathrm{PE}$ in colleges and universities so that students can shift their attention to sports and exercise. This study measured student ratings of VRT by students in five schools, compared the number of students who exercised automatically before and after the sport's reform, and analyzed the impact of VRT on students. Students' evaluation of VRT is conducted in the form of scoring, with an overall score of 10. Finally, the average scores of students on VRT are measured. Table 4 shows the statistical results and the innovation evaluation results of the number of students who independently engage in PE and sports.

According to the data in Table 4, we can see that after the five schools have used VRT in PE, the number of students who independently engage in PE and sports is increasing, and students have higher ratings for VRT. We convert the 
Table 1: Data table related to sports safety incidents.

\begin{tabular}{lcccc}
\hline Colleges and universities & Before use & After use & Usage (\%) & Dwindle numbers (\%) \\
\hline$A$ & 55 & 12 & 25 & 78.2 \\
$B$ & 51 & 13 & 21 & 74.5 \\
$C$ & 43 & 15 & 35 & 65.1 \\
$D$ & 66 & 10 & 30 & 85 \\
$E$ & 68 & 14 & 79.4 \\
\hline
\end{tabular}

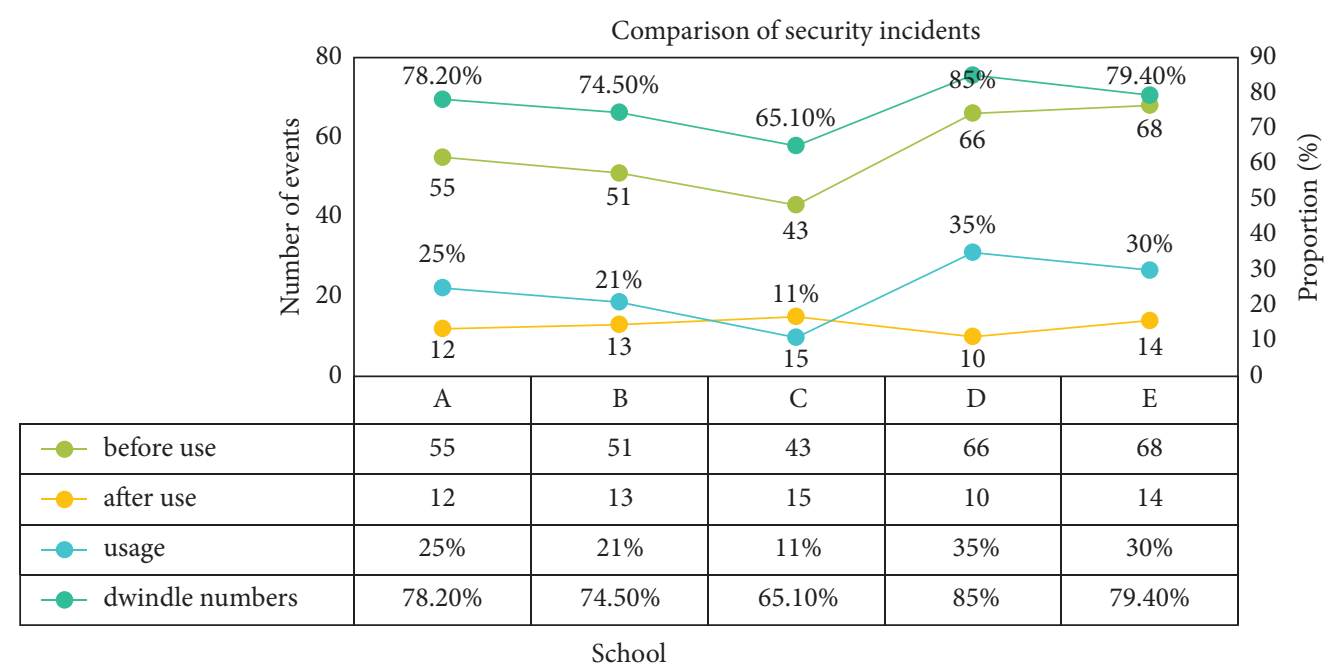

FIGURE 1: A comparison chart of sports safety incidents in colleges and universities.

TABLE 2: Number of teachers in each impact item.

\begin{tabular}{|c|c|c|c|c|c|c|}
\hline & Philosophy & Attitude & Content & Method & Management & Evaluation \\
\hline$A$ & 76 & 65 & 54 & 70 & 34 & 54 \\
\hline$B$ & 68 & 32 & 57 & 64 & 42 & 67 \\
\hline$C$ & 71 & 48 & 34 & 51 & 36 & 51 \\
\hline$D$ & 65 & 59 & 60 & 63 & 38 & 63 \\
\hline E & 63 & 62 & 52 & 42 & 31 & 66 \\
\hline
\end{tabular}

TABLE 3: Teacher ratio table for each impact item.

\begin{tabular}{ccccccc}
\hline & Philosophy (\%) & Attitude (\%) & Content (\%) & Method (\%) & Management (\%) & Evaluation (\%) \\
\hline$A$ & 32.6 & 27.9 & 23.2 & 30 & 14.6 & 16.7 \\
$B$ & 27.1 & 12.7 & 22.7 & 25.5 & 16.1 & 23.2 \\
$C$ & 31.7 & 21.4 & 15.2 & 22.8 & 26.7 & 22.8 \\
$D$ & 27.5 & 25 & 25.4 & 26.7 & 16.1 & 26.7 \\
$E$ & 26 & 25.6 & 21.5 & 17.4 & 12.8 & 27.3 \\
\hline
\end{tabular}

data in Table 4 into the form of graphs to better observe the changes in the data. The conversion results are shown in Figure 4.

According to the data in Figure 4, we can see that five schools have used VRT in PE to have a certain impact on students. From the perspective of the growth rate, the number of students in school $D$ who independently engage in PE and sports has increased the most, with an increased ratio of 0.412 , and the number of students in school $C$ who independently engage in sports learning and sports has the least increased, with an increase of 0.205. Judging from the students' ratings of VRT, the students' ratings of VRT are all above 7 points, indicating that the experience of VRT to students is not bad, but it needs improvement.

We also sampled 100 students' interest in sports learning and sports exercise and divided the interest into four levels: very interested (I), generally interested (II), not interested (III), and very uninterested (IV). The number of students in 


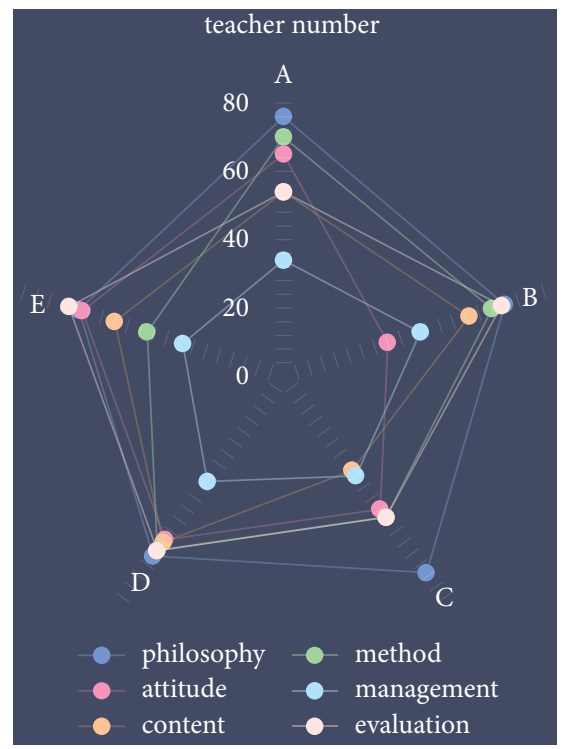

FIgURE 2: The number of teachers in each impact item.

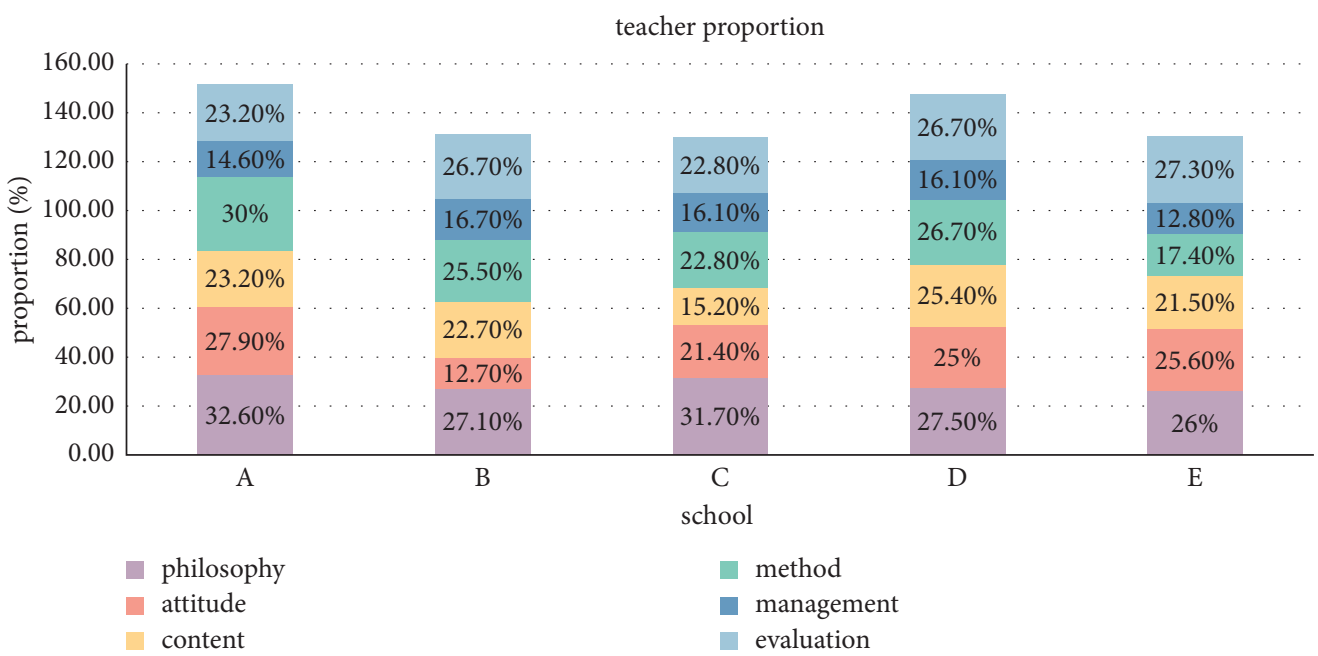

FIgURE 3: The proportion of teachers in each impact item.

TABLE 4: Student population statistics and innovation evaluation table.

\begin{tabular}{lcccc}
\hline & Before use & After use & Growth rate & Average score \\
\hline$A$ & 1870 & 2531 & 0.353 & 8 \\
$B$ & 1644 & 2036 & 0.238 & 7.6 \\
$C$ & 1636 & 1971 & 0.205 & 7.5 \\
$D$ & 1868 & 2637 & 0.412 & 8.2 \\
$E$ & 1892 & 2614 & 0.382 & 8.7 \\
\hline
\end{tabular}

each level was counted, and the change of interest of sports students before and after the use of VRT was analyzed. Finally, the number of students in each grade is shown in Table 5.

According to the data in Table 5, we can see the change in students' interest in sports learning and sports. The data in Table 5 are transformed into the form of a graph for observation, and the influence of VRT on students' interests is analyzed. The conversion result is shown in Figure 5.
According to the data in Figure 5, we can see that before the use of VRT in PE in five schools, the number of students who are not interested in sports learning and sports is about 30. After using VRT in PE teaching, the number of students who are not interested in sports learning and sports is greatly reduced, and the number of students is about 20. The number of students who are very interested in sports learning and sports before is only 15 at most. After the 
TABLE 5: Change of students' interest.

\begin{tabular}{ccccccccc}
\hline & \multicolumn{3}{c}{ Before use } & & \multicolumn{3}{c}{ After use } \\
& I & II & III & IV & I & II & III \\
\hline$A$ & 12 & 53 & 20 & 15 & 35 & 47 & 10 & IV \\
$B$ & 10 & 61 & 18 & 11 & 29 & 57 & 14 \\
$C$ & 7 & 54 & 25 & 24 & 17 & 65 & 15 \\
$D$ & 15 & 62 & 20 & 3 & 45 & 52 & 3 \\
$E$ & 13 & 60 & 18 & 9 & 39 & 56 & 3 \\
\hline
\end{tabular}

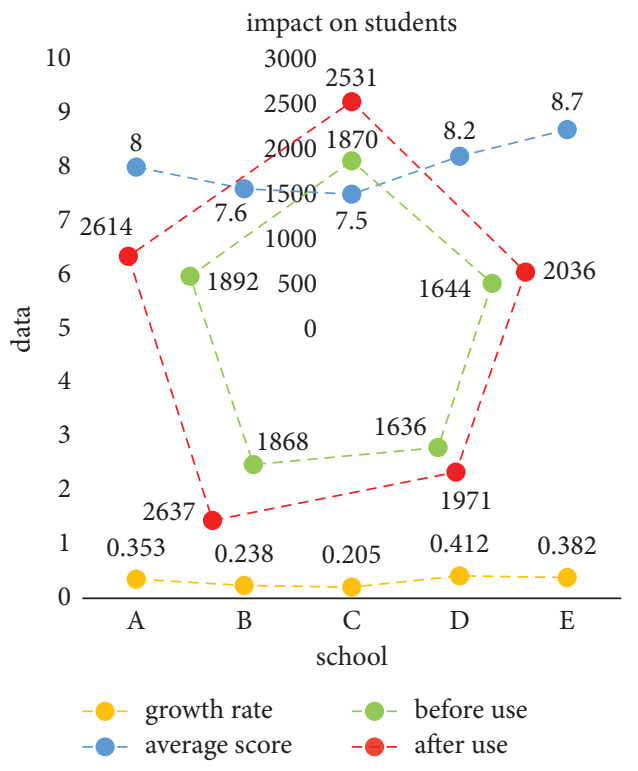

FIgURE 4: Changes in the number of students and evaluation graph.

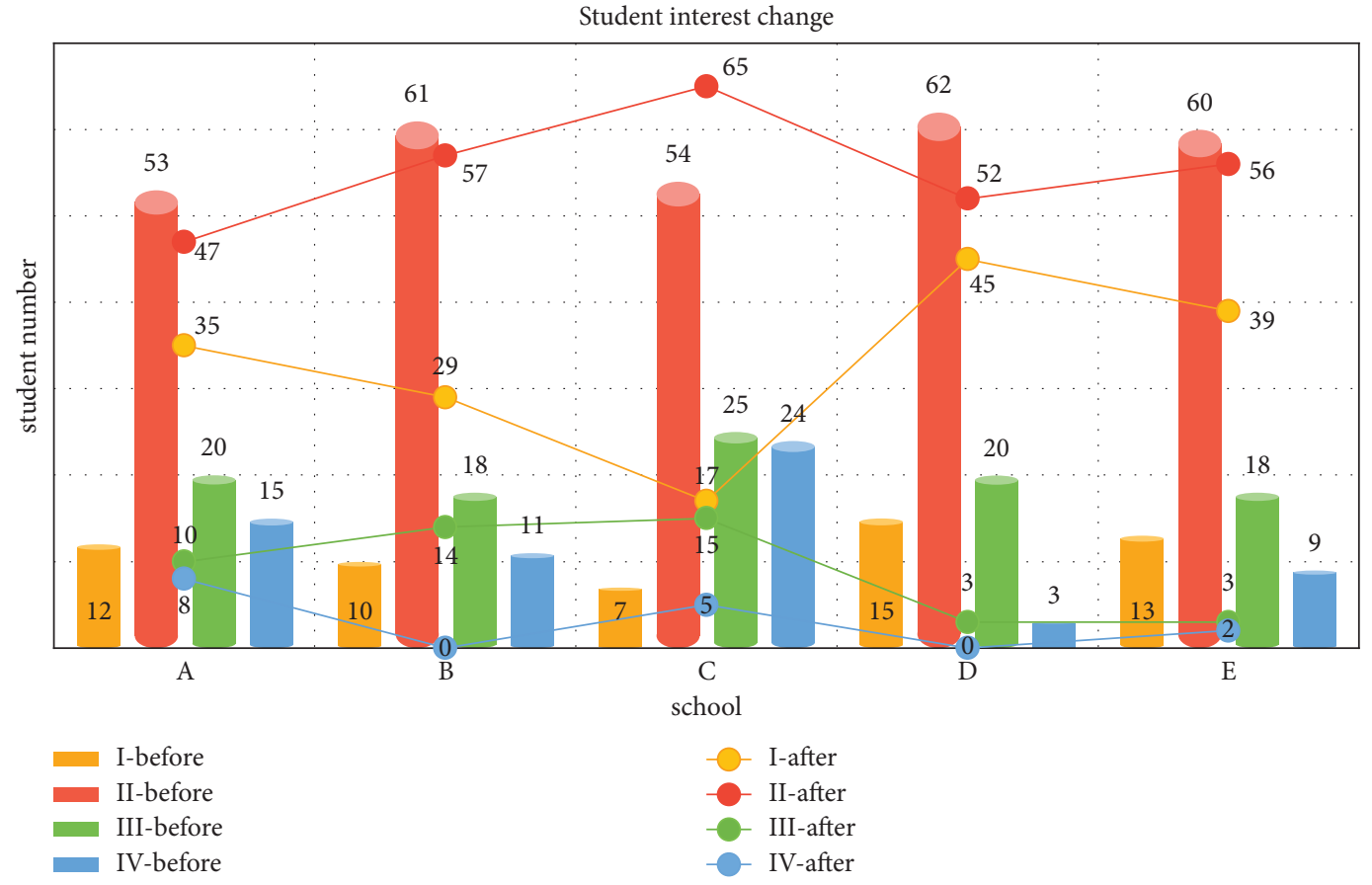

Figure 5: Change of students' interest. 
TABLE 6: The application proportion of each technology in PE and teaching.

\begin{tabular}{|c|c|c|c|c|c|}
\hline Teaching mode & $A(\%)$ & $B(\%)$ & $C(\%)$ & $D(\%)$ & $E(\%)$ \\
\hline VR & 25 & 21 & 11 & 35 & 30 \\
\hline AI & 3 & 5 & 2 & 5 & 5 \\
\hline $\mathrm{MC}$ & 10 & 10 & 5 & 6 & 9 \\
\hline $\mathrm{TT}$ & 45 & 44 & 70 & 37 & 36 \\
\hline APP & 17 & 20 & 12 & 19 & 20 \\
\hline
\end{tabular}

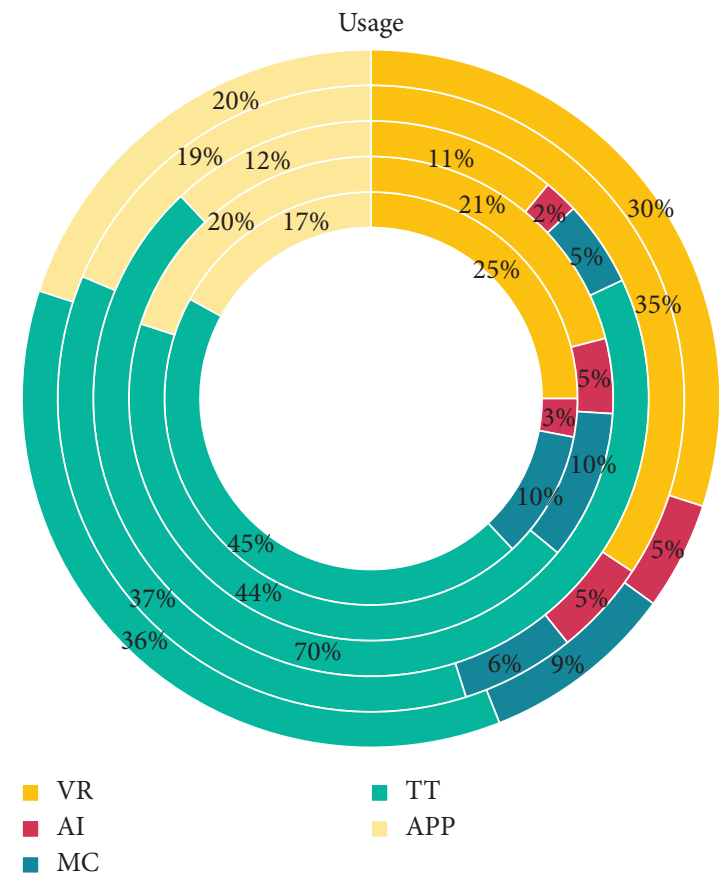

FIGURE 6: Proportion of VRT and other teaching modes in college PE.

application of VRT, the number of students in this interest level increases rapidly, and the number of schools with the largest number of students reaches 45 . Therefore, the application of VRT in PE can improve students' interest in sports learning and sports.

4.4. Application of VRT and Other Teaching Modes in PE. This paper investigates the application of VRT (VR) in the PE teaching of the five schools and makes statistics on the application proportion of artificial intelligence (AI), media courseware (MC), traditional teaching (TT), and application program (APP) in PE and teaching. The final statistical results are shown in Table 6.

According to the data in Table 6, we can see the application of VRT, artificial intelligence, media courseware, traditional teaching, and application programs in PE College teaching. This percentage data usage is the use of various teaching modes in five schools using accounting; usage amount represents the number of classes using some teaching model; the total class number represents the total number of all classes of the school, using the formula that can be expressed as

$$
\text { usage }=\frac{\text { usage amount }}{\text { total class number }} \times 100 \% .
$$

In order to observe the application of these teaching modes more clearly, we converted the data in Table 6 into graph form, and the final result is shown in Figure 6.

According to the usage ratio chart in Figure 6, we can know the application of various teaching modes in PE and teaching in five schools. The rings in the ring diagram from the inside to the outside, respectively, represent schools $A, B$, $C, D$, and $E$. The proportion of the application of VRT in PE of five schools is $25 \%, 21 \%, 11 \%, 35 \%$, and $30 \%$, respectively. It can be seen that the application of VRT in PE teaching of five schools is in the middle position, and there is still room for development.

\section{Conclusions}

In recent years, the Education Bureau and the leaders of colleges and universities have attached great importance to the PE level of students and the teaching conditions of PE teachers. In order to enable students to make extensive progress in morality, intelligence, mathematics, and 
aesthetics, the university's teaching model is constantly improving and changing with each passing day. In the Internet of Things environment, researchers have tried to apply $5 \mathrm{G}$ technology and VRT to the teaching of various university subjects to improve the teaching methods of university education, and good progress has been made.

The research report combines the research content of previous scientists. The development of the Internet of Things is inseparable from the support of 5G technology, uses VRT to study college sports, and applies VRT to college sports. The experiment counted the number of sports safety incidents that occurred before and after the application of VRT in five universities, collected and analyzed the teaching data of PE teachers in five universities, and studied the changes in student learning and interest in sports. Before and after the use of VRT in these five universities, the changes in the number of students participating in sports were measured. Finally, the use of VRT and several other teaching methods in these five universities was measured. The experiment increased students' interest in participating in sports schools and sports. The theory and technical mastery of virtual reality technology is not thorough enough, and there is no comprehensive thinking and understanding in the application. In the research, there is no more in-depth, detailed, and sufficient investigation and research on physical education that requires the application of virtual reality technology.

\section{Data Availability}

The data that support the findings of this study are available from the corresponding author upon reasonable request.

\section{Conflicts of Interest}

The author declares no conflicts of interest with respect to the research, authorship, and/or publication of this article.

\section{Acknowledgments}

The authors would like to acknowledge the 2018 National Education Ministry Humanities and Social Science Youth Fund Project: Research on the "Sports and Medicine Integration" Talent Training Model under the Background of Healthy China, under Project No. 18YJC890027.

\section{References}

[1] Z. Lv and K. S. Amit, "Big data analysis of internet of things system," ACM Transactions on Internet Technology (TOIT), vol. 21, no. 2, 2020.

[2] W. Yang, "On college physical education teaching reform based on "students physical health standard"," Journal of Inner Mongolia University of Finance and Economics (Comprehensive Edition), vol. 013, no. 003, pp. 138-140, 2015.

[3] J. Zhang and Z. Zhang, "Research on the reform strategy of college physical education under the background of internet+"Internet+"," Journal of Hebei University of Engineering (Social Science Edition), vol. 036, no. 001, pp. 98-100, 2019.
[4] Y. U. Wei-Ping and L. Jin-Song, "Rethinking the reform of college physical education courses in the New Era," Journal of Heb Sport University, vol. 1, no. 1, pp. 35-37, 2019.

[5] S. Stormoen, H. B. Urke, H. E. Tjomsland, W. Bente, and D. Åge, "High school physical education: What contributes to the experience of flow?" European Physical Education Review, vol. 22, no. 3, pp. 44-45, 2016.

[6] J. L. Maples-Keller, B. E. Bunnell, S.-J. Kim, and B. O. Rothbaum, "The use of virtual reality technology in the treatment of anxiety and other psychiatric disorders," Harvard Review of Psychiatry, vol. 25, no. 3, pp. 103-113, 2017.

[7] M. Koeva, M. Luleva, and P. Maldjanski, "Integrating spherical panoramas and maps for visualization of cultural heritage objects using virtual reality technology," Sensors, vol. 17, no. 4, pp. 829-831, 2017.

[8] D. Guido, H. Song, and A. Schmeink, Big Data Analytics for Cyber-Physical Systems: Machine Learning for the Internet of Things, Elsevier, Amsterdam, Netherlands, pp. 1-360, 2019.

[9] J. Q. Coburn, I. J. Freeman, and J. L. Salmon, "A review of the capabilities of current low-cost virtual reality technology and its potential to enhance the design process," Journal of Computing \& Information Science in Engineering, vol. 17, no. 3, pp. 1-15, 2017.

[10] L. Li, F. Yu, D. Shi et al., "Application of virtual reality technology in clinical medicine," American Journal of Tourism Research, vol. 9, no. 9, pp. 3867-3880, 2017.

[11] C. Donghui, L. Guanfa, Z. Wensheng, L. Qiyuan, B. Shuping, and L. Xiaokang, "Virtual reality technology applied in digitalization of cultural heritage," Cluster Computing, vol. 22, no. 4, pp. 1-12, 2017.

[12] Y. Sang, Y. Zhu, H. Zhao, and M. Tang, "Study on an interactive truck crane simulation platform based on virtual reality technology," International Journal of Distance Education Technologies, vol. 14, no. 2, pp. 64-78, 2016.

[13] S. A. Alo, Y. Jiazi, C. Thomas, and M. T. William, "A study of LoRa: Long range \& low power networks for the internet of things," Sensors, vol. 16, no. 9, p. 1466, 2016.

[14] C. Aranzazu-Suescun and M. Cardei, "Distributed algorithms for event reporting in mobile-sink WSNs for internet of things," Tsinghua Science and Technology, vol. 22, no. 4, pp. 413-426, 2017.

[15] C. Chang, S. N. Srirama, and R. Buyya, "Mobile cloud business process management system for the internet of things: A survey," ACM Computing Surveys, vol. 49, no. 4, pp. 1-42, 2016.

[16] H. Song, G. A. Fink, and S. Jeschke, Security and Privacy in Cyber-Physical Systems: Foundations, Principles and Applications, pp. 1-472, Wiley-IEEE Press, Chichester, UK, 2017.

[17] M. Agiwal, N. Saxena, and A. Roy, "Towards connected living: $5 \mathrm{G}$ enabled internet of things (IoT)," IETE Technical Review, vol. 36, no. 2, pp. 190-202, 2019.

[18] H. Sun, X. Wang, R. Buyya, and J. Su, "CloudEyes: cloudbased malware detection with reversible sketch for resourceconstrained internet of things (IoT) devices," Software: Practice and Experience, vol. 47, no. 3, pp. 421-441, 2017.

[19] S. Li, L. D. Xu, and S. Zhao, "5G internet of things: a survey," Journal of Industrial Information Integration, vol. 10, no. 1, pp. 1-9, 2018.

[20] A. H. Sodhro, S. Pirbhulal, S. Arun Kumar, S. Lohano, G. H. Sodhro, and Z. Luo, "5G-Based transmission power control mechanism in fog computing for internet of things devices," Sustainability, vol. 10, no. 4, pp. 1258-1259, 2018. 
[21] M. D. Giudice, "Discovering the internet of things (IoT) within the business process management," Business Process Management Journal, vol. 22, no. 2, pp. 263-270, 2016.

[22] M. R. Palattella, M. Dohler, A. Grieco et al., "Internet of things in the 5G Era: Enablers, architecture, and business models," IEEE Journal on Selected Areas in Communications, vol. 34, no. 3, pp. 510-527, 2016.

[23] J. Liu, R. Shangguan, X. D. Keating, J. Leitner, and Y. Wu, "A conceptual physical education course and college freshmen's health-related fitness," Health Education, vol. 117, no. 1, pp. 53-68, 2017.

[24] J. Zhu, "Exploration of college physical education reform based on MOOC," Journal of Jiaying University, vol. 035, no. 011, pp. 97-100, 2017.

[25] H. Y. Tsai, L. C. Chang, and C. C. Yeh, "An analysis of high school physical education teachers' teaching behaviors," Eurasian Soil ence, vol. 42, no. 3, pp. 326-333, 2016.

[26] Y. Sun, L. Liu, and X. Du, "Study on the reform of sports fitness program in college physical education curriculum," Heilongjiang Science, vol. 009, no. 008, pp. 52-53, 2018.

[27] L. Tang and S. Zhang, "The optimization of college physical education teaching quality based on cloud theory and prospect theory," Journal of Zhaoqing University, vol. 040, no. 005, pp. 77-82, 2019.

[28] S. R. Phillips, K. Mercier, and S. Doolittle, "Experiences of teacher evaluation systems on high school physical education programs," Physical Education and Sport Pedagogy, vol. 22, no. 4, pp. 1-14, 2017. 